\title{
Medioambiente pleistocénico y ocupaciones humanas en el valle medio del río Magdalena, Colombia
}

Pleistocene Environment and Human Occupations in the Middle Valley of the Magdalena River, Colombia

\section{RESUMEN}

Este artículo presenta una revisión de los trabajos disponibles sobre las ocupaciones humanas tempranas y las condiciones medioambientales en el Magdalena Medio. Nos enfocamos en la temporalidad de la transición del Pleistoceno al Holoceno con el fin de evaluar la potencialidad de la región para albergar sitios con evidencias del uso del paisaje por parte de sociedades de cazadores recolectores. Para lograr esto, caracterizamos la zona, elaboramos el contexto paleoambiental y relacionamos los sitios arqueológicos, con lo cual aportamos a la generación de hipótesis sobre la dispersión humana durante ese período.

Palabras clave: paleoambiente, pleistoceno final, ocupación temprana, Valle Medio del río Magdalena.

\section{Abstract}

This article presents a review of the available work on early human occupations and environmental conditions in the middle valley of the Magdalena river. The focus is on the temporality of the transition from the Pleistocene to the Holocene epoch in order to assess the potential of the region to house sites with evidence of landscape use by collecting hunter societies. To achieve this, the area was
Mario Alonso

Bermúdez-Restrepo

Profesor / Investigador del área de Arqueología en el Departamento de Antropología y Sociología de la Universidad de Caldas. Estudiante de Doctorado en FACSO, Universidad $\mathrm{Na}$ cional del Centro de Provincia de Buenos Aires. Coordinador del Laboratorio de Arqueología de la Universidad de Caldas y el grupo GIGA

$\square$ mario.bermudez@ucaldas.edu.co

(1) ORCID: 0000-0002-2135-3353

$\checkmark$ Google Scholar 
characterized, the paleoenvironmental context was elaborated, and the archaeological sites were related, thereby contributing to the generation of hypotheses about human dispersion during the period.

Keywords: paleoenvironment, final Pleistocene, early human occupation, Middle Magdalena River Valley.

\section{Introducción}

$\mathrm{D}$ esde el inicio de las investigaciones arqueológicas en el norte del continente suramericano, el valle del río Magdalena, por su posición y características geográficas, fue considerado por los investigadores como una de las rutas de poblamiento desde el norte después de sobrepasar los corredores centroamericanos (ReichelDolmatoff, 1978; Correal, 1988; Ardila y Politis, 1989). En el presente siglo, tanto las evidencias artefactuales, compuestas principalmente por artefactos líticos tallados, como las fechas radiocarbónicas, los datos paleoambientales disponibles y las evidencias de megafauna, permiten elaborar hipótesis sobre una ocupación que se extiende más allá del Holoceno, poniendo en discusión el paradigma Clovis (Aceituno y Rojas-Mora, 2015). Incluso algunas evidencias indirectas en el continente pueden indicar que bandas de Homo sapiens con un modo de producción de forrajeo, estuvieron presentes desde el último máximo glaciar en diferentes ambientes, desde las costas y las sabanas hasta las montañas andinas. Las huellas de uso en abrigos rocosos, cuevas y campamentos al aire libre sustentan, con toda seguridad, la ocupación humana de Suramérica desde el Younger Dryas (Correal y Van der Hammen, 2001; Dillehay et al., 2008; Fiedel, 2000; Sheinsohn, 2003).

Un análisis de los diferentes proxies climáticos y ambientales, demuestra que los mosaicos medioambientales de finales del pleistoceno fueron aptos para los movimientos poblacionales de Homo por todos los continentes, incluyendo el suramericano. Se expone en este artículo una compilación de datos que nos acercan al ambiente que pudieron encontrar las bandas de primeros pobladores del norte del continente suramericano, especialmente al sur de la cuenca media del río Magdalena, en Colombia. 


\section{Geología y geomorfología en la zona sur del valle medio del río Magdalena}

El Valle del río Magdalena es el resultado del levantamiento tectónico causado por la presión convergente de las placas de Nazca y Sudamericana. Es un valle alargado orientado en dirección sur-norte que separa las Cordilleras Central y Oriental de Colombia. Su proceso de formación se remonta a los comienzos del Mesozoico, momento desde el cual ha pasado por diversas etapas que lo han formado, al principio de tipo distensivo, y más tardíamente, en el Cenozoico, de tipo compresivo (Mojica y Franco, 1990).

La zona de interés se encuentra ubicada en el sector sur de la región del Magdalena Medio caldense ${ }^{1}$, debido a que el grupo de investigación que el autor coordina, viene realizando una evaluación geoarqueológica en la que se vinculan varios trabajos de grado de antropología y dos trabajos de tesis de la maestría en ciencias de la tierra, en los cuales se han detectado varias paleosuperficies disponibles en temporalidades más tardías. En este sector se encuentran los fondos de valles transversales, que irrigan la cuenca principal, la mayor parte de ellos de corta distancia y bajo caudal, a excepción de los ríos Gualí y Guarinó que corren desde el macizo volcánico de la cordillera Central. En esta zona, "la tectónica se expresa en fallas normales cuya evolución ha causado adelgazamientos y contrastes de las distintas formaciones mesozoicas y cenozoicas" (Guzmán, com. per., 2016²).

El piedemonte occidental del valle del río Magdalena, a la altura de los municipios de Mariquita y Honda, en el Tolima y La Dorada y Victoria, en Caldas, cerca de la cuenca baja del río Guarinó, se encuentra sobre cuatro formaciones geológicas predominantes (figura 1). La primera de esquistos, cuarcitas y mármoles con inclusiones regionales de migmatitas y anfibolitas de edad paleozoica, situada en las vertientes finales. La segunda de rocas sedimentarias conglomeráticas de diferente grano, como areniscas y arcillolitas de edad miocénica, denominada como grupo Honda. La tercera compuesta principalmente por conglomerados, areniscas y pocas limolitas y arcillolitas de composición tobácea, en la cual los fragmentos que componen los conglomerados

\footnotetext{
Específicamente el sitio de interés en el programa de investigación que adelanta el grupo GIGA de la Universidad de Caldas y que ha sido escogido como sitio de trabajo de campo en el $V$ Taller de Geoarqueología, se referencia entre los $5^{\circ} 17^{\prime}$ y $5^{\circ} 25^{\prime}$ de latitud norte y entre los $74^{\circ} 40^{\prime}$ y $74^{\circ} 44^{\prime}$ de longitud oeste.

Carlos Guzmán es profesor de estratigrafía y sedimentología en el departamento de Geología de la Universidad de Caldas.
} 
son de origen extrusivo dacítico y andesítico; todo el conjunto de edad terciaria (Plioceno), las cuales conforman la formación Mesa y suprayace a la anterior. Finalmente, depósitos cuaternarios semiconsolidados o no consolidados en las vegas de los ríos y quebradas, que son resultado en principio de una relativa estabilidad geotectónica y de las inundaciones repentinas causadas por los deshielos del final de la glaciación, ambas sumadas a los procesos finales de deposición por parte de las fuentes tributantes del río (IGAC, 1985).

Los depósitos finales son terrazas disectadas que se sitúan en la zona plana del valle, las cuales se registran en el mapa geológico de Colombia como Q-al, depósitos aluviales (zona blanca cerca del río en la imagen 1), $y$ Q-t, terrazas aluviales (zona amarilla que rodea al río, en la misma imagen). En una escala más detallada se presentan tres niveles topográficos de edad cuaternaria, que fueron depositados por las paleofuentes, en algunos casos retrabajadas por las fuentes actuales. Las terrazas Qt0, Qt1 y Qt2 están diferenciadas por su altura, composición petrográfica y el grado de consolidación; las más antiguas y altas (Qt2), fueron y son talladas o remodeladas por los procesos geomorfológicos de erosión laminar y remoción por escorrentías (Castañeda y Martínez, 1998). Los modelamientos erosivos hicieron que algunas de estas se vieran en el pasado como colinas bajas, redondeadas o con cimas planas, aptas para el asentamiento, cuando se encontraban cerca de las fuentes hídricas permanentes. Estas geoformas son usadas actualmente para el pastoreo extensivo, la ubicación de asentamientos y cultivos de maíz, centeno y pasto con irrigación. Los depósitos de vega están situados en los cauces permanentes y en los efímeros, siguiendo las líneas de escorrentía. Se componen de materiales finos a muy finos no consolidados (desde gravas hasta arcillas), que por la dinámica aluvial sufren procesos de transporte y deposición continuos. En estas vegas inundables son colonizadas por pastos y rastrojos, resistentes a los procesos propios de esta posición geomorfológica. 


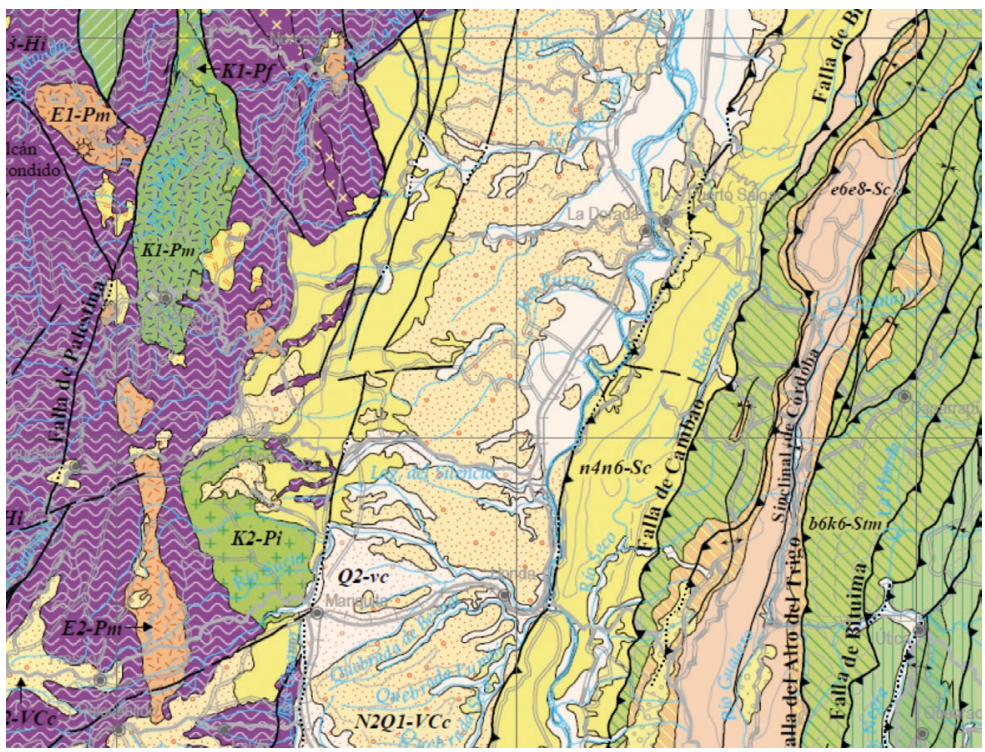

Figura 1. Geología del área de referencia tomada del mapa geológico de Colombia (IGAC, 2015).

Los suelos en la zona en mención son derivados de depósitos fluviales, pero los demás actores formadores influyen su génesis, lo cual hace que en el mapa de suelos se clasifiquen como fluvents, que indican su insipiencia, pero además se presentan suelos de diferentes órdenes como inceptisoles, aridisoles y alfisoles. En general son suelos poco productivos.

El área presenta en la actualidad un clima muy cálido, ya que se encuentra por debajo de los $500 \mathrm{~m}$ de altura sobre el nivel del mar, se clasifica como semihúmedo por la precipitación y la evapotranspiración (Corpocaldas, 1997). La zona de vida predominante es de bosque húmedo tropical (bh T), aunque existen zonas muy áridas con pequeños parches que, en los inviernos prolongados, avanzan sobre los pastizales. Las precipitaciones muestran dos épocas marcadas de lluvias entre finales de marzo y principios de junio y entre septiembre y noviembre, las demás épocas se consideran secas, mostrando un pico elevado de bajas precipitaciones en los meses de febrero y julio, aunque se presentan lluvias torrenciales esporádicas (Castañeda y Martínez, 1998). Cuando estas precipitaciones sobrepasan los niveles normales, en los ciclos de 'La Niña', generan inundaciones en las terrazas Qt0, que retransportan y redepositan materiales, lo cual afecta tanto los procesos cumúlicos como los pedogenéticos y por ende los posibles sitios ribereños. 


\section{El contexto ambiental del Pleistoceno Final}

Hacía el Pleistoceno final, la mayor parte de la conformación macroescalar geológica y geomorfológica del valle medio del río Magdalena presentaba una imagen bastante parecida a la actual. El levantamiento tectónico, el vulcanismo y los procesos erosivos de gran magnitud ya habían esculpido el paisaje, dejando expuesto un valle amplio de laderas alargadas, cañones transversales escarpados en $\mathrm{V}$ que siguen las fuentes de agua permanentes, y una llanura de inundación, relativamente plana, a poca altura sobre el nivel del mar, en la que discurría la paleofuente en un curso entre meándrico y recto. Hoy en día hacía el sur del área en mención, las fracturas causadas por la presión tectónica se expresan en el cambio de rumbo del cauce del río y los raudales de Honda, más al oeste, sobre la vertiente occidental, en dirección sur-norte, las evidencias de la presión se manifiestan en la falla de Mulatos (Castañeda y Martínez, 1998).

En el transcurso de la última época glaciar, los cambios climáticos fluctuaron desde muy fríos (estadiales) a unos menos fríos (interestadiales), sin alcanzar temperaturas ni temporalidades que los puedan clasificar como interglaciares. Para el final de la glaciación hubo un último enfriamiento global, conocido como último máximo glaciar (UMG o LGM por sus iniciales en inglés), ocurrido entre 23 y 19 mil años antes del presente, con algunas variaciones locales bien marcadas (Bush et al., 2009). Este enfriamiento afectó más fuertemente las latitudes altas, con mayor contundencia las situadas en el hemisferio norte debido a la masa continental, pero las variaciones siguen en discusión ya que los datos en algunos casos no son muy coincidentes (Lowe y Walker, 2015).

Las consecuencias del UMG fueron variadas, especialmente si se relacionan con la expansión de la especie humana (Bourgeon, Burke y Higham, 2017). El aumento de acumulación de hielos en los mantos Laurentino y Finoescandinavo, y en las altas montañas tropicales y subtropicales, generó una disminución del nivel del mar, que alcanzó los 130 a 140 metros por debajo del nivel actual, producto de la evaporación del agua y su no regreso por el ciclo natural; así, la "tierra firme" ocupada por el hielo en el norte y en los macizos continentales, se compensó con tierra expuesta en los litorales de otras latitudes, en los que la plataforma continental es relativamente poco profunda, lo que generó la apertura de puentes naturales entre continentes e islas y la expansión areal, entre otros del Caribe y la Antártida (Grobe, Huybrechts y Fûterer, 1993).

En Centroamérica y el norte de Suramérica, una cota del nivel del mar por debajo de 130 metros, produjo un cambio drástico en el paisaje, 
exponiendo una línea intermareal y una llanura costera mucho más retiradas de las cordilleras. A medida que las nuevas tierras continentales fueron expuestas, experimentaron el avance del proceso de colonización de la masa biótica, lo que amplió los mosaicos medioambientales de las tierras bajas. De otro lado, la línea mareal que sirve de nivel base para los ríos, hizo que la curva de gradiente de los cauces aumentara considerablemente, lo cual activó la dinámica geomorfológica, generando procesos de excavación en los fondos de los valles y cambios en la conformación de las estructuras de las cuencas, como en el caso de las evidencias fluviales del río Magdalena a la altura de la depresión momposina (Herrera, Sarmiento, Romero, Botero y Berrío, 2001).

Otro cambio significativo que pudo influir sobre los movimientos poblacionales y de colonización de los seres humanos en el norte de Suramérica, tiene que ver con la distribución de los mosaicos vegetales. Las evidencias polínicas muestran que las coberturas boscosas en las cordilleras migraron. A medida que el nival invadía las cimas altas de las cordilleras, el páramo, los bosques andino y subandino se movieron hasta $1500 \mathrm{~m}$ vertiente abajo, mientras que los fondos de los valles de baja altura, como los interandinos de los ríos Magdalena, Cauca y Atrato, permanecieron con vegetación de bosque tropical, más o menos húmedo (Hooghiemstra y Rahn, 1994; Marchant et al., 2002).

Hacía el 19 o 20 mil antes del presente, se inició el proceso de deglaciación. No es claro todavía si comenzó en el hemisferio norte y un poco después en el hemisferio sur, o viceversa (Lowe y Walker, 2015). Tampoco son claras sus causas, hay diferentes hipótesis: desde los ciclos de Milankovitch, la disminución del albedo y la banquisa marina, los cambios en la circulación de los vientos y los efectos de los gases de invernadero (CO2, CH4, N2O y H2O en vapor) (Saltzmank y Stievenard, 1999). Este calentamiento, que aceleró el ciclo del agua, tuvo efectos directos en el nivel del mar, el cual fue incrementándose gradualmente hasta alcanzar los niveles actuales en aproximadamente 10 mil años, es decir a inicios del Holoceno (Clark, Marshall, Mix y Weaver, 2004; Alley, Clark, Huybrechts y Joughin, 2005).

Es de resaltar que, al momento del inicio de la deglaciación, inmediatamente después del UMG, hace aproximadamente 19 mil años, ya se encontraban en la cuenca del Magdalena grupos de cazadores-recolectores (Correal y Van der Hammen, 2001), estas evidencias son coherentes con los datos reportados de otros sitios en Suramérica como El Jobo, Monteverde, Pedra Furada y Patagonia, como se menciona adelante. 


\section{Las condiciones del Younger Dryas en el norte de Suramérica}

El proceso de deglaciación en el hemisferio norte que, como se dijo, inició en el 19 mil antes del presente y que se prolongó en el período Bølling-Allerød, se detuvo en el 12,9 mil por diferentes causas, retomando condiciones frías por más de 1,3 mil años (Bradley, 1999; Rincón y Martínez, 2004). Los datos en diferentes latitudes coinciden con un momento de enfriamiento y desecamiento, aunque en la Antártida los resultados obtenidos de las columnas de hielo indican un calentamiento progresivo (Grobe, Huybrechts y Fûterer, 1993; Lowe y Walker, 2015).

En el norte de Suramérica los resultados de los análisis en las columnas de polen, en el mismo tiempo en el que está ocurriendo el Younger Dryas, indican que este período se caracteriza por ambientes más abiertos que los actuales en las alturas medias y bajas de las cordilleras, también indican que el cambio del mosaico vegetal no es tan abrupto como se pensaba (Marchan et al., 2002). Los datos informan que los mosaicos medioambientales en las partes altas de las cordilleras fueron muy afectados: el nival que en el UMG se extendía hasta los 3000 metros de altura, al inicio del Holoceno apenas alcanzaba los 3800 metros, igualmente el ambiente de páramo que ocupaba alturas entre los 2000 y los 3000 metros durante el interestadial de Guantiva y el estadial del Abra (Van der Hammen y Hooghiemstra, 1995), retrocedió remontando las cordilleras hasta alturas desde los 2800 y 3800 en el inicio del Holoceno; por el contrario el bosque tropical apenas tuvo un cambio de 100 metros entre los 800 y 900 si comparamos ambos períodos (Flantua, Hooghiemstra, Van Boxel, Cabrera, González-Carranza y González-Arango, 2014).

Finalmente, los resultados de análisis de polen sostienen la hipótesis de que en alturas medias y altas de las cordilleras Oriental y Central, ocurrió un enfriamiento de hasta $-6 \mathrm{C}^{\circ}$, bajo condiciones más secas, en la temporalidad de los 11 mil a 9,5 mil que coincide con las condiciones del Younger Dryas europeo (Van der Hammen y Hooghiemstra, 1995). 


\section{Los sitios arqueológicos del Pleistoceno Final y el Holoceno Temprano en Suramérica}

Desde la década del 50 del siglo XX, después del descubrimiento del C14, distintos sitios arqueológicos con fechas que sobrepasan el límite Pleistoceno - Holoceno, fueron reportados en diferentes latitudes del continente suramericano, desde el istmo de Panamá hasta la Tierra del Fuego. Contextos arqueológicos como el Lago Madden en Panamá, El Abra y Tequendama en Colombia, Taima Taima y El Jobo en Venezuela, El Inga en Ecuador, Pikimachay y Cueva Guitarrero en Perú, Monte Verde y Tagua Tagua en Chile, Cueva Fell y Los Toldos en Argentina, y Pedra Furada en Basil, entre otros muchos demostrarían la presencia humana desde finales del Pleistoceno.

Aunque la ocupación de estos sitios estaba bien documentada y en muchos casos asociada a fauna extinta, la mayor parte de aquellos que sobrepasaban edades holocénicas tuvieron contradictores académicos que prefirieron las conservadoras fechas asociadas a la tradición Clovis, pero a finales del siglo XX casi una centena de sitios en Suramérica reportaban fechas pleistocénicas, tanto en los ambientes andinos como costeros y de sabanas, e incluso en lugares donde hoy se ubican las selvas tropicales densas (Dillehay et al., 2008; Goebel, Waters y O'Rourke, 2008). Para mencionar solo algunos se destacan los siguientes y se grafican en la figura 2.

El sitio de Pubenza (Correal y Van der Hammen, 2001), cuya edad radiocarbónica se remonta a 16550+150 14C AP. (calibrada a años calendario de 19 mil años AP). Las Vegas (Stothert, 2011), con fechas que alcanzan los 13820 y 12950 años calibrados AP. Los contextos de Lagoa Santa (Araujo et al., 2012) tales como Santana do Riacho fechado en $9460 \pm$ 11014C AP (calibradas a años calendario en 11155 a 10481 AP) y el sitio Lapa Grande de Taquaracu con una edad radiocarbónica entre $9990 \pm 6014 \mathrm{C}$ AP. y $9900 \pm 6014$ C AP (calibradas entre 11750 y 11210 años calendario AP). Finalmente, el sitio de Monte Verde en Chile, con una temporalidad de $12,290 \pm 60$ C14 yr B.P., calibradas a años calendario en 14,6 mil años AP (Dillehay, 2008).

Como complemento, según datos obtenidos en la Amazonía (Van der Hammen y Absy, 1994), durante el período en mención predominaba un clima más seco que haría cambiar los mosaicos medioambientales; la proporción entre las sabanas, la selva tropical y los ambientes costeros sería muy diferente de la actual, lo que permitiría los movimientos poblacionales con mayor facilidad. En las tierras bajas la extensión 
de la sabana se impuso sobre la selva tropical húmeda, e incluso pudo generar, en las áreas más secas, paisajes arídicos, campos de dunas y depósitos eólicos, y en las tierras altas la expansión de las nieves perpetuas y los bosques de páramo también fue significativa como vimos ya. Este comportamiento ambiental pudo ser el resultado de los movimientos de la zona de confluencia intertropical (ZCI).

Con este panorama geográfico, las evidencias encontradas en más de una veintena de sitios fechados entre el tardiglaciar y el inicio del Holoceno en el norte de Suramérica (como se muestra en la imagen 2) y teniendo en cuenta que la subida del nivel del mar se prolongó hasta el Holoceno temprano, con el consabido pulso del Younger Dryas (Alley et al., 2005), como se expuso atrás, se debe entender que muchos de los sitios arqueológicos paleoindios, o de edad pleistocénica, deben estar hoy a alturas que fueron cubiertas por los océanos Pacífico y Atlántico cerca de las plataformas costeras, y sepultados por sedimentos fluviales y de vertiente en las áreas continentales, lo que implicaría su parcial o total destrucción por los fenómenos geomorfodinámicos y su subrepresentación, como se propone en otra parte (Bermúdez, 2010). También debe tenerse en cuenta que los corredores disponibles para los movimientos poblacionales tendrían que calcularse con la totalidad de las tierras emergidas por el fenómeno de baja en el nivel del mar, además de la reconstrucción total de las coberturas vegetales y los mosaicos medioambientales.

Con base en la distribución de los sitios y en los resultados de investigaciones sobre los paleoambientes dominantes a finales del Pleistoceno, autores como Lanata et al. (2008), Surovell (2003) y Dillehay (2002), han propuesto modelos generales de poblamiento del continente americano y suramericano, tomando como punto de partida el ingreso por el estrecho de Bering; otros investigadores consideran que los datos paleoclimáticos, paleoecológicos y genéticos, tanto en esta temporalidad como en temporalidades posteriores (Holoceno Medio), no son del todo coherentes (Grosjean et al., 2003; Kitchen, Miyamoto y Mulligan 2008; Faught, 2008). 


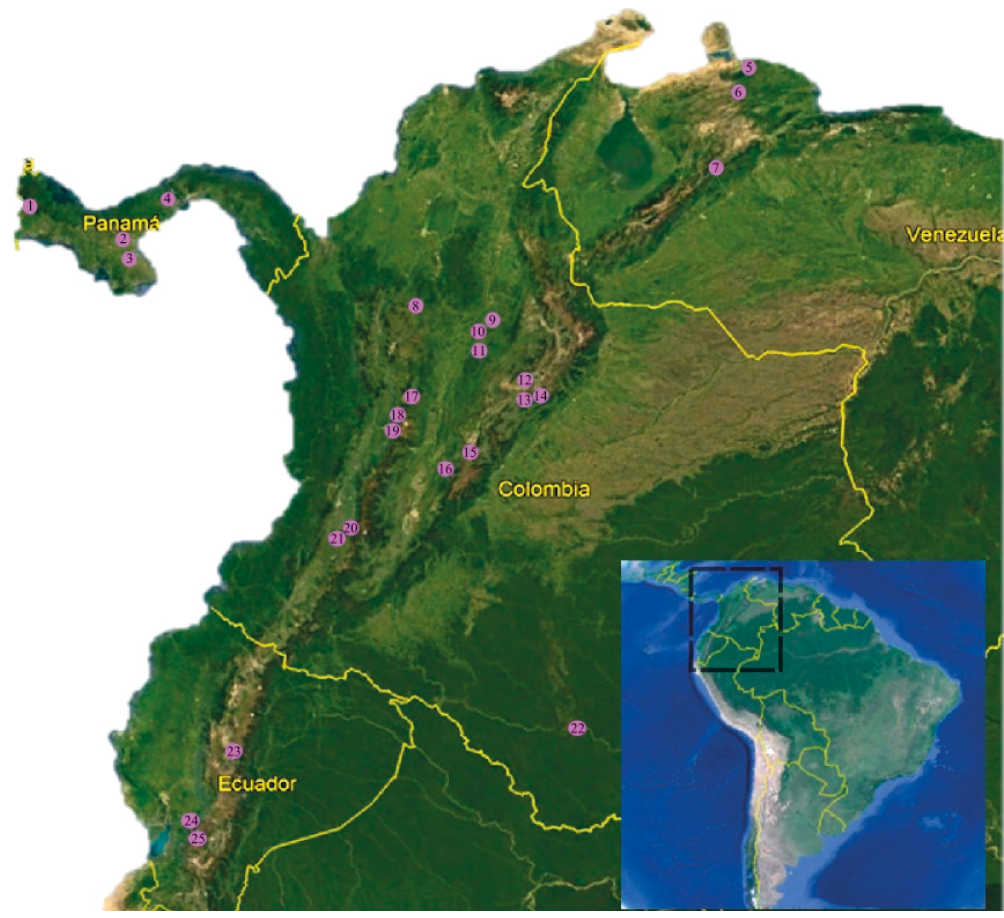

Figura 2. Ubicación de sitios fechados entre el Pleistoceno Final y el Holoceno Temprano en el Norte de Sudamérica: 1. Turrialba 2. La Yeguada 3. Los Vampiros. 4 Lago Maden. 5. Taima Taima 6. El Jobo. 7. El Vano. 8 Porce. 9- Yondó. 10. La Palestina; 11- San Juan de Bedout. 12. El Abra. 13. Tequendama.14. Tibitó. 15. El Totumo. 16. Pubenza 17.El Jazmín 18. Cuba. 19. La Mikela. 20. La Elvira. 21. San Isidro. 22. Araracuara. 23. El Inga.; 24. Cubilán 25. Chobshi

Aun así, como se ha venido argumentando, las condiciones ecológicas en el continente suramericano, y en general en toda América a finales del Pleistoceno, incluyendo el UMG y el YD, fueron propicias para que los grupos humanos sacaran a relucir las ventajas adaptativas que acumularon durante milenios en las condiciones adversas de la era glaciar, las cuales tenían que ver con la flexibilidad en el modelo de captación de los recursos (economía de subsistencia basada en una dieta amplia) y la organización sociocultural (bandas de tipo familiar de baja densidad muy móviles). La nube de puntos que representa a los sitios arqueológicos fechados en el tardiglaciar, demuestra que las estrategias de los cazadores recolectores (forrajeros eclécticos) fueron exitosas desde las costas oceánicas, hasta las altas montañas y desde las latitudes medias hasta las ecuatoriales (Dillehay, Ramírez, Pino, Collins, Rossen, y Pino-Navarro, 2008).

Finalmente, se puede afirmar que el conjunto de sitios arqueológicos reportados en la transición pleistoceno holoceno en el norte de Suramérica indica que los movimientos poblacionales en este período fueron continuos 
y muy probablemente rápidos, debido a la oferta ambiental (entre nichos, parches y refugios) y la adaptabilidad humana para ocuparlos, explotarlos, compartirlos o defenderlos. También se puede concluir que la densidad poblacional pudo ser mayor de la pensada: las dinámicas climáticas, en las que se incluyen los cambios del nivel del mar, temporadas de lluvias anormales que generan erosión y sedimentación, las geomorfodinámicas en las vertientes y las costas, que producen movimientos en masa capaces de modelar el paisaje y remodelar las superficies, el vulcanismo activo en este período, que pudo tanto remover, como sepultar, sitios completos bajo mantos de tefras profundas, generar lahares o desolación por caída de cenizas, pueden estar sub-representando significativamente las ocupaciones humanas.

\section{Los sitios de fechas pleistocénicas en el Magdalena Medio}

\section{Perspectivas}

Al hacer un inventario de los sitios con edad pleistocénica, nos damos cuenta que para el valle medio del río Magdalena, estos están representados únicamente por el sitio arqueológico de Pubenza (Correal y Van der Hammen, 2001), el cual no ha sido reconocido totalmente por la comunidad académica internacional. Los otros sitios antiguos, fechados en la transición entre el Pleistoceno y el Holoceno en Colombia y el norte de Suramérica (imagen 2), al final del Estadial del Abra, que es coincidente con el Younger Dryas europeo, serían los reportados en la zona norte de la región en cuestión, principalmente en la vertiente oriental de la cordillera Central (López, 2008) y en el área de captación de la cuenca en la sabana de Bogotá (Correal, 1988). Otro conjunto de sitios, un poco más numerosos se reportan en toda la región, pero estos podrían considerarse como del Holoceno Temprano (Aceituno y Rojas-Mora, 2015; López, 2008).

Para un mejor entendimiento de estos problemas es necesario refinar los procesos investigativos y trabajar en equipos transdisciplinarios con objetivos comunes. Un detallado muestreo y control de los proxies para la reconstrucción medioambiental, permitirá a futuro aumentar la resolución, desde la búsqueda hasta la interpretación, más cuando el problema incluye la adaptación y adaptabilidad humanas a los cambios naturales de diferente escala y a los propiciados por su propio actuar.

En este último sentido, problemas de investigación abordados desde una perspectiva amplia que incluya la reconstrucción paleoambiental en temporalidades recientes, y no tan recientes, que se enmarcan desde el final del Pleistoceno y en todo el Holoceno, deben tener en cuenta el 
aumento de la densidad de población, a partir de los procesos de experimentación, manejo y domesticación de plantas y los consabidos cambios en los modos de producción, y el hacinamiento después de la "revolución urbana", fenómenos que han afectado las dinámicas ambientales a nivel planetario, y que muchos de los procesos-respuesta sistémicos de hoy, pueden ser el resultado de la toma de decisiones adaptativas de nuestra especie en el pasado.

\section{Referencias}

Aceituno, J. y Rojas-Mora, E. (2015). Lithic technology studies in Colombia during the late Pleistocene and early Holocene. Chungara, Revista de Antropología Chilena, 47, (1)

Alley, R., Clark, P., Huybrechts, P. y Joughin, I. (2005). Ice-Sheet and Sea-Level Changes. Science, 310

Ardila, G. y Politis, G. (1989). Nuevos datos para un viejo problema. Boletín del Museo del Oro, (23), 3-45.

Araujo, A., Neves, W. y Kipnis, R. (2012). Lagoa Santa revisited: an overview of the chronology, subsistence, and material culture of paleoindian sites in eastern central Brazil. Latin American Antiquity, 23(4), 533-550.

Bermúdez, M. (2010). Dinámicas geomorfológicas de piedemonte y procesos de transformación de sitios arqueológicos en el Magdalena Medio caldense. Virajes, 12...

Bourgeon, L., Burke, A. y Higham. T. (2017). Earliest Human Presence in North America Dated to the Last Glacial Maximum: New Radiocarbon Dates from Bluefish Caves, Canada. PLoS ONE, 12 (1): e0169486. doi:10.1371/journal.pone.0169486

Bradley, R. (1999). Paleoclimatology. Reconstructing Climates of the Quaternary. Second Edition. Academic Press, An Imprint of Elsevier. San Diego, CA.

Bush, M., Correa-Metrio, A., Hodell, D., Brenner, M., Anselmetti, F., Ariztegui, D. Mueller, A., Curtis, J., Grzesik, D., Burton, C. y Gilli, A. (2009). Re-evaluation of Climate Change in Lowland Central America During the Last Glacial Maximum Using New Sediment Cores from Lake Petén Itzá, Guatemala. In F. Vimeux et al. (eds.), Past Climate Variability in South America and Surrounding Regions. Developments in Paleoenvironmental Research 14.

Castañeda, A y Martínez, F. (1998). Evaluación preliminar por inundación del río Magdalena en La Dorada. Tesis de grado. Universidad de Caldas. Manizales.

Clark, P., Marshall, A., Mix, A. y Weaver, A. (2004). Rapid Rise of Sea Level 19,000 Years Ago and Its Global Implications. Science, 304(5674), 1141-1144.

Corpocaldas. (1997). Plan integral de manejo distrito de manejo integrado madrevieja de Guarinocito. Diagnostico socioeconómico y ambiental. Informe Final.

Correal, G. (1988). Las culturas más antiguas de Colombia. Estadio de cazadores y recolectores. Historia de Colombia, Salvat Ed. Bogotá. 
Correal, G. y Van der Hammen, T. (2001). Mastodontes en un humedal pleistocénico en el valle del Magdalena (Colombia) con evidencias de la presencia del hombre en el pleniglacial. Boletín de Arqueología, (16), 1.

Dillehay, T. (2002). Climate and Human Migrations. Science, 298.

Dillehay, T. (2008). Profiles in Pleistocene History. In Handbook of South American Archaeology. Silverman H, and Isbell, W. editors. Springer.

Dillehay, T., Ramírez, C., Pino, M., Collins, M., Rossen, J. y Pino-Navarro, J. (2008). Monte Verde Seaweed, Food, Medicine, and the Peopling of South America. Science, 320.

Faught, M. (2008). Archaeological Roots of Human Diversity in the New World: A Compilation of Accurateand Precise Radiocarbon Ages from Earliest Sites. American Antiquity, 73(4) pp. 670-698.

Fiedels. (2000). The Peopling of the New World: Present Evidence, New Theories, and Future Directions. Journal of Archaeological Research, (8), 1.

Flantua, S., Hooghiemstra, H., Van Boxel, J., Cabrera, M., González-Carranza, Z y GonzálezArango, C. (2014). Connectivity dynamics since the last glacial maximum in the northern Andes a pollen-driven framework to assess potential migration. In Paleobotany and Paleografy...

Goebel, T., Waters, M. y O'Rourke, D. (2008). The Late Pleistocene Dispersal of Modern Humans in the Americas. Science, 319.

Grobe, H., Huybrechts, P. y Fûterer, D. (1993). Late Quaternary record of sea levels changes in the Antartic. Geol Rundsch.

Grosjean, M., Cartajena, I., Geyh, M. y Núñez, L. (2003). From proxy data to paleoclimate interpretation. The mid-Holocene paradox of the Atacama Desert, northern Chile. Palaeogeography, Palaeoclimatology, Palaeoecology, 194.

Hemming, S. (2004). Heinrich events massive late Pleistocene detritus layers of the north atlantic and their global climate imprint. Review of Geophysics, 42.

Herrera, L., Sarmiento, G., Romero, F., Botero, P. y Berrío, J. (2001). Evolución ambiental de la depresión momposina (Colombia) desde el Pleistoceno Tardio a los paisajes actuales. Geología Colombiana, 26, Bogotá.

Hooghiemstra, H., y Ran, E. (1994). Upper and Middle Pleistocene climatic change and forest development in Colombia: pollen record Funza II (2-158 m core interval) Palaeogeography, Palaeoclimatology, Palaeoecology, 109.

IGAC. (1985). Características geográficas de Caldas. Prensa Nacional. Bogotá.

IGAC. (2015). Mapa geológico de Colombia. Versión digital. Bogotá.

Johnson, A. y Earle, T. (2003). La Evolución de las Sociedades Humanas. Desde los grupos cazadores - recolectores al Estado agrario. Ariel Prehistoria. Barcelona.

Kitchen, A., Miyamoto, M. y Mulligan, C.J. (2008). A Three-Stage Colonization Model for the Peopling of the Americas. PLoS ONE 3(2): e1596. doi:10.1371/journal.pone.0001596

Lanata, J., Martino, L., Osella, A. y García-Herbst, A. (2008). Ambiente y demografía durante la dispersión humana inicial en Sudamérica. En López C y Ospina G. (Comp.) Ecología Histórica. Interacciones Sociedad - Ambiente a distintas escalas socio - temporales. UTP. Pereira. 
López, C. (2008). Landscape development and the evidence for early human occupation in the Inter-Andean tropical lowlands of Magdalena river, Colombia. Syllaba Pres. E-Book.

Lowe, J. y Walker, M. (2015). Reconstructing quaternary environments. Third edition. New York.

Marchant, R., Behling, H., Berrio, J.-C., Cleef, A., Duivenvoorden, J., Hooghiemstra, H., Kuhry, P., Melief, B., Schreve-Brinkman, E., Van Geel, B., Van Der Hammen, T., Van Reenen, G. y Wille, M. (2002). Pollen-based biome reconstructions for Colombia at 3000, 6000, 9000, 12 000, 15000 and $1800014 \mathrm{C}$ yr ago: Late Quaternary tropical vegetation dynamics. J. Quaternary Sci., 17.

Mojica, J. y Franco, R. (1990). Estructura y evolución tectónica del valle Medio y Superior del Magdalena, Colombia. Geología Colombiana, 17, p. 41-64.

Reichel-Dolmatoff, G. (1978). Colombia Indígena: Período Prehispánico. Manual de historia de Colombia. Tomo 1. Procultura. Bogotá.

Saltzmank, E. y Stievenard, M. (1999). Climate and atmospheric history of the past 420,000 years from the Vostok ice core. Antarctica Nature, 399.

Sheinsohn, V. (2003). Hunter-Gatherer archaeology in South America. Annual Review of Anthropology.

Surovell, T. (2003). Simulating coastal migration in New World colonization in current anthropology. Volume 44, Number 4, August-October.

Van der Hammen, T. y Hooghiemstra, H. (1995). The El Abra Stadial, a Younger Dryas Equivalent in Colombia. Quaternary Science Reviews, 14.

Van der Hammen, T. y Absy, M. (1994). Amazonia during the last glacial. Palaeogeography, Palaeoclimatology, Palaeoecology, 109. 